\title{
Apply Particle Swarm Optimization Algorithm to Measure the Software Quality
}

\section{Ibrahim Ahmed Saleh}

i.hadedi@gmail.com

College of Computer Sciences and Mathematics

Salha Raa'd Mahammead

salhamohammedab@gmail.com
Received on: 29/01/2018

Accepted on: 20/05/2018

\begin{abstract}
The process of improvement software quality began from early stages of software engineering development. It uses multiple quality metrics which are very important in software development. To calculate the standards quality of in software testing has been adopted. The software testing is focusing on the Software defect. In this paper is proposed new methods which combine the particle swarm optimization (PSO) to handle the best features Extraction with back-propagation networks to testing and evaluation of the data set. The paper depended database for NASA standards data. The result and experiment method improved quality performance for all classification methods used in the research"Combining Particle Swarm Optimization based Feature Selection and Bagging for Software Defect Prediction."
\end{abstract}

Keywords: Software Defect, Particle Swarm Optimization, Neural Network Back Propagation, NASA.

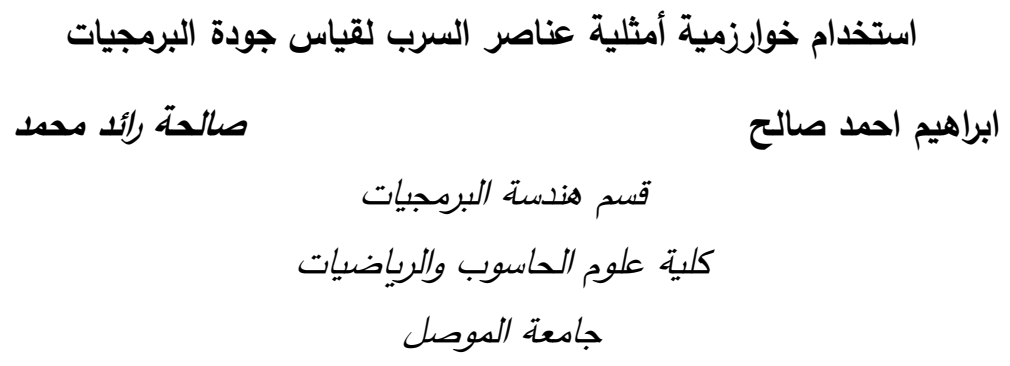

تاريخ قبول البحث: 2018105120

تاريخ استلام البحث: $2018 \backslash 01129$

\section{الملخص}

بدأت عملية تحسين الجودة للبرمجيات من مراحل مبكرة في تطوير هندسة البرمجيات; وذلك باستخدام مقاييس الجودة التي تعد مهمة جداً في عملية تتمية البرمجيات، وقد اعتمد حساب مقاييس الجودة في اختبار

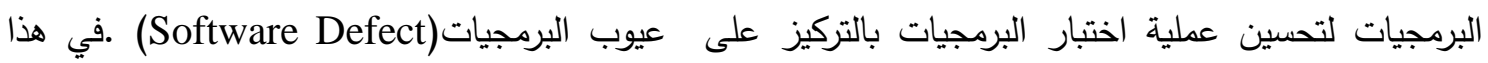
البحث اقتراح نظام برمجي يجمع بين خوارزمية سرب الطيور (Particle Swarm Optimization) في استخلاص الخواص للتعامل مع أفضل المميزات واستخدام الشبكات العصبية ذات الانتشار العكسي( Neural (Network Back Propagation مقاييس وكالة ناسا (NASA)، وقد بيّنت النتائج أنَّ الطريقة المستخدمة قد حققت تحسناً ملحوظاً في عملية أداء

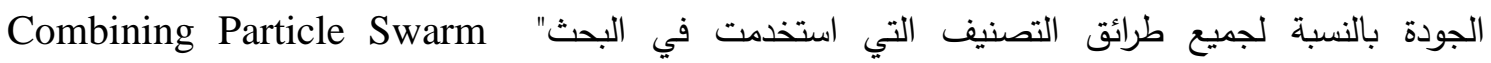

." Optimization based Feature Selection and Bagging for Software Defect Prediction الكلمات المفتاحية: عيوب البرمجيات، خوارزمية سرب الطيور، الانتشار العكسي للشبكة العصبية، ناسا. 
تعد عملية بناء برمجيات عالية الجودة للبرمجيات مهمة صعبة جداً من حيث عدد مكوناتها الأساسية

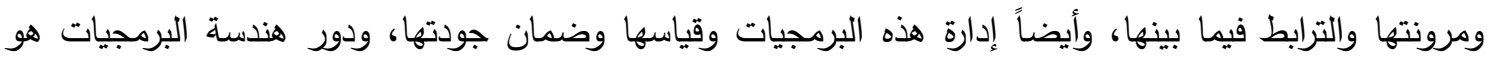

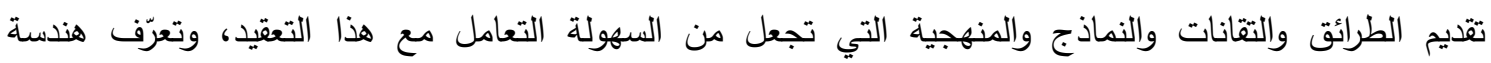
البرمجيات وفق Institute of Electrical and Electronics Engineers: "هندسة البرمجيات بأنها تطبيق المنهجية والنظامية والنهج القابل للقياس الكمي لتطوير البرمجيات وتتفيذها وصيانتها، أي بمعنى تطبيق الهندسة

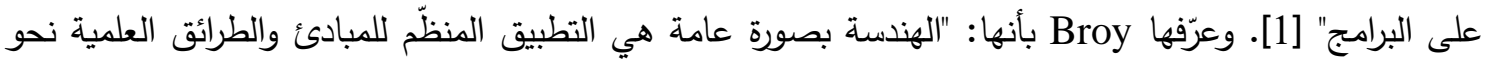
بناء كفوء وفعّال للهياكل والمكائن، ومن ناحية هندسة نظم البرمجيات فإن الهدف الجوهري هو تحسين القدرات

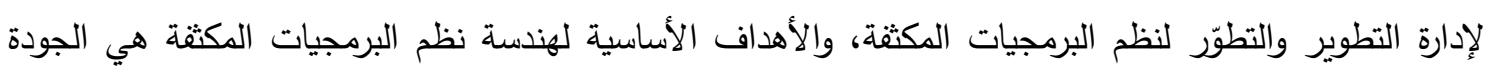

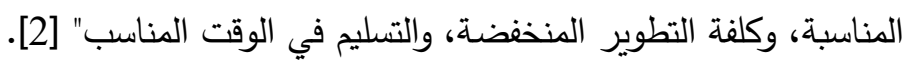

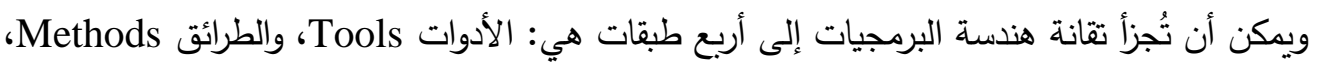

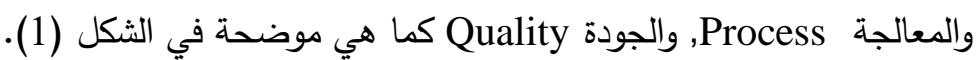

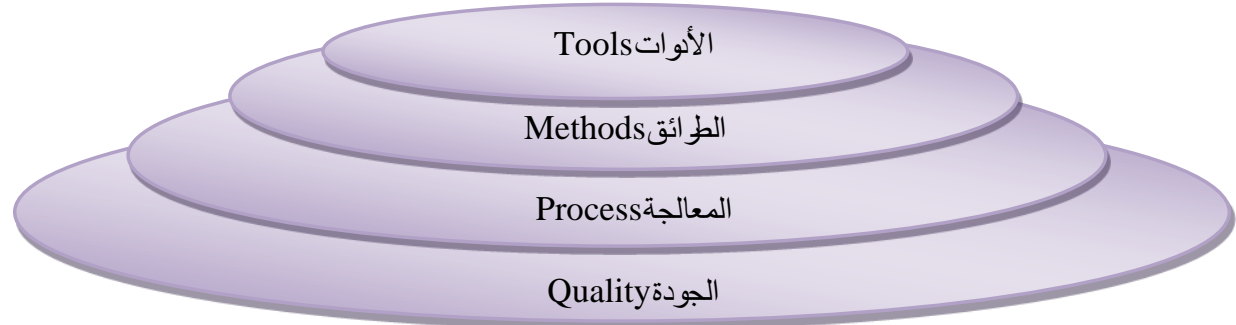

الشكل (1): المبادئ الاساسية لهندسة البرمجيات

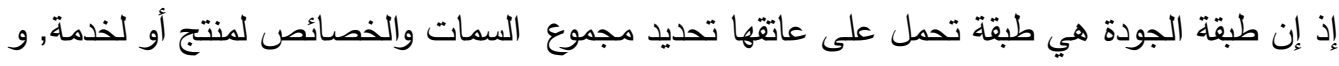

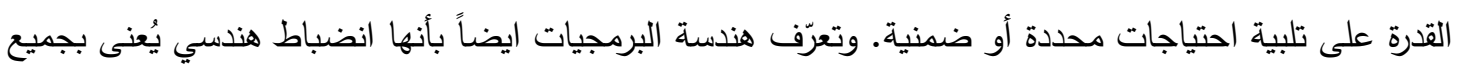

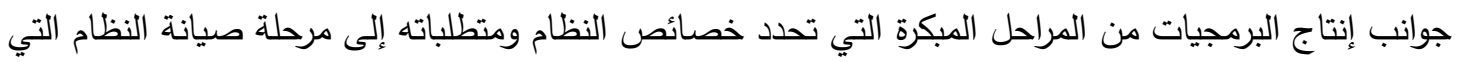

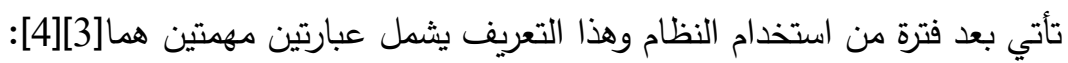

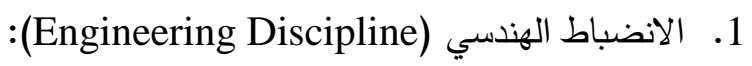

يتضمن تطبيق النظريات والطرائق والأدوات حيثما تكون مناسبة وبطريقة انتقائية تتتاسب مع حل المشكلة

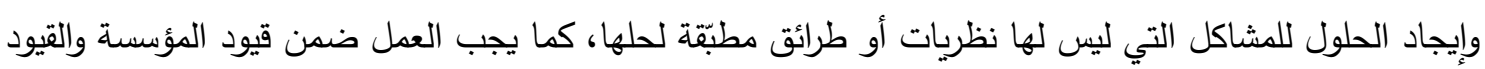
المالية وإيجاد الحلول ضمن تلك القيود. 2. جميع جوانب إنتاج البرمجيات (All aspects of software production) : هندسة البرمجيات ليست معنية بالعمليات التقانية لتطوير البرمجيات فحسب بل مسؤولة أيضاً عن فعاليات أخرى مثل إدارة المشاريع وتطوير الأدوات والنظريات والطرائق لدعم إنتاج البرمجيات.

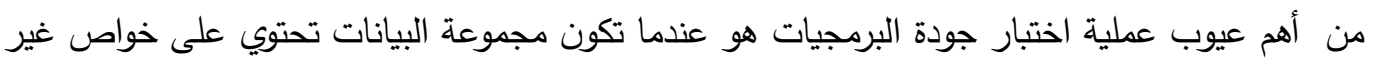
موثوقة [5] . على أي حال، فإن هذا التشويش في مجموعة البيانات قد يؤدي إلى استعمالات خاطئه باستخدام الطرائق التقليدية; لذا خاصية استخلاص الخواص(feature selection) عادة ما تستخدم في عملية تقليل البيانات واختيار بيانات ذات سمات عالية وصاخبة، وتستخدم خوارزميه استخلاص الخواص لتحديد العناصر المعينة مابين الصحيح والأكثر صحة التي تساعد الباحثين من إيجاد أفضل الحلول التي تتراوح قيمها ما بين الأمثل 
وأقصى الامثل والتي تساعد على تقليل مساحة البحث والتعقيد الحسابي ، ونتيجة لذلك، شبه الأمثل للحلول المثلى

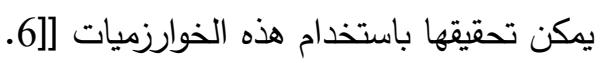
إن عملية تحقيق الأكثر صحة هو لإيجاد أفضل حل مثالي في فضاء البحث واستخدام البحث الكامل, ويعمل على زيادة القدرة لايجاد الحلول العالية النوعية على النحو ملحوظ ضمن مدة زمنية معقوله, وتتضمن عملية

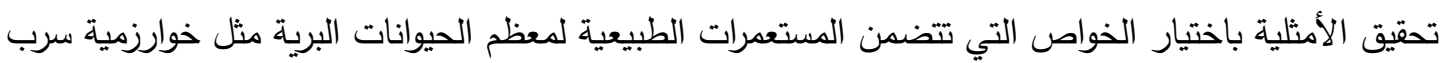

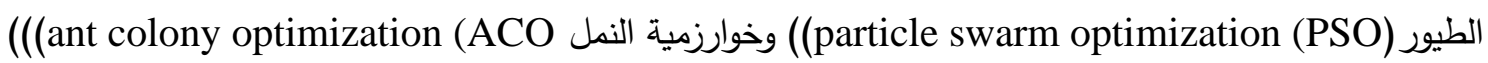
وغيرها[7].

في هذا البحث اعتمد موضوع جودة البرمجيات; لأنه موضوع مهم لهندسة البرمجيات التي يتخصص بها قسمنا, وقد استخدامت طريقة جديدة للجمع بين خوارزمية سرب الطيور المثالية (PSO) والثبكة العصبية ذات الانتشار العكسي (Neural Network Back Propagation) لفحص جودة البرمجيات ودقتها ، إذ اعتمدت خوارزمية الأسراب لاستخلاص المميزات, وتستخدم الثبكة العصبية للتعامل مع مشكلة عدم التوازن الطبقة. تستخدم الثبكة بسبب فعالية في التعامل مع اختلال الطبقة وتقييم الطريقة المقترحة باستخدام أحدث مجموعات البيانات

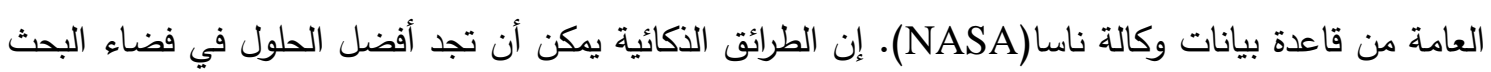

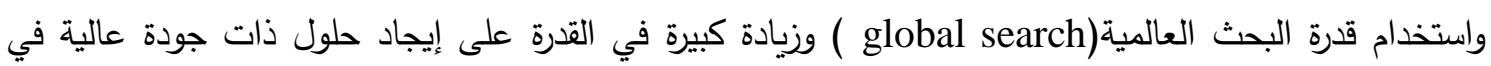
غضون مدة معقولة من الزمن[7].

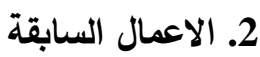

حاول الباحث غريغور بانفوسكي في عام 2008 [8] إجراء دراسة لتقييم جودة منتج البرمجيات, فقد كانت دراسته تركز على تقييم جودة الخصائص الخارجية لمنتج البرمجيات التي تمثل تقييم السلوك لكنتج البرمجيات أثناء تنفيذه. وتم التركيز في هذه الدراسة على تطوير أنموذج الجودة ( 9126ISO/IEC) على معايير البرمجيات, وأجريت هذه الدراسة على 7 عينات لمنتج البرمجيات واستعمال معايير أنموذج وتوصل الباحث الى أن خصائص جودة المنتج الخارجية يمكن اعتمادها كفئة أو مجال, وأن المعايير التي يوفرها 2-9126ISO/IEC يتطلب جعلها متكيفة مع خصائص منتج البرمجيات على النحو مناسب.

\section{3. جودة البرمجياتQuality Software}

عرف رونان [9] جودة البرمجيات بأنها امتداد للتعريف الصناعي الذي يضم مجموعة من الميزات المرغوب

فيها التي ينبغي إدراجها في المنتج وذلك لتحسين أدائه مدى الحياة، وبعبارة اخرى هو التحسين المستمر للمنتج

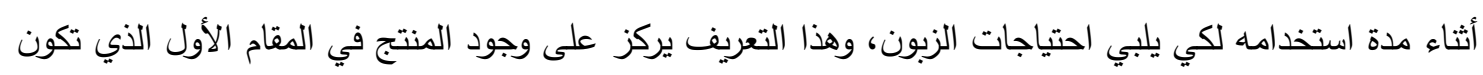

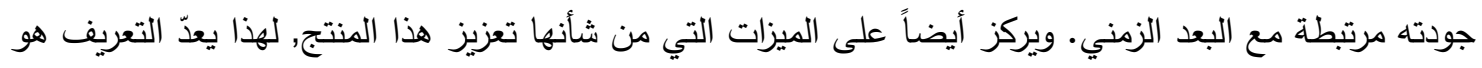
الأثمل من ناحية تطور جودة المنتج أثناء مدة استخدامه.

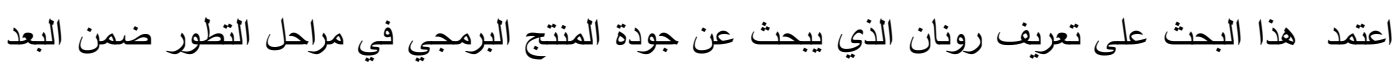
الزمني, وهذا يتماشى مع أساليب تطوير البرمجيات الحديثة التي تكون بشكل حلقات متزايدة لمراحل التطوير، وفي كل حلقة نستطيع قياس الجودة على نحو جيد, وذلك لأن المنتج في أية مرحلة تطويرية يمكن أن يسلم كاملاً على لئل

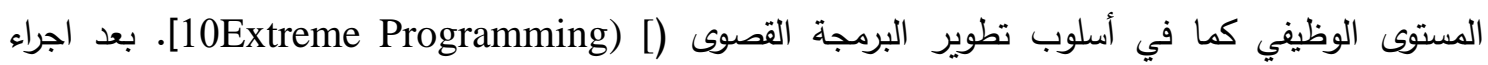


الاختبار والانتهاء من عملية التطوير يجب أن يعرف مدى جودة البرمجيات التي ستسلم. إذ يعتد على الفرق بين البيانات الأساسية والبيانات بعد الانتهاء من عمليات الاختبار والتطوير .

4. الخوارزمية المقترحة في البحث:

1.4. خوارزمية سرب الطيور(PSO)

منذ أكثر من خمسة عقود أثبت العلماء وجود أنواع متعددة من التقانات الذكائية التي تستوحي من

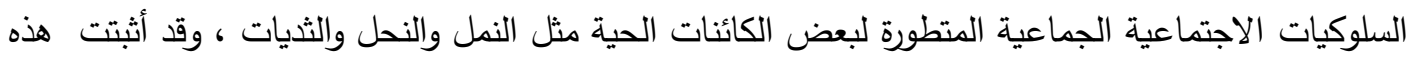
الخوارزميات فعاليتها - كما هي في الطبيعة - وكان لها دور كبير من التطبيقات الرائعة والفعالة التي شملت شتى لئى المجالات.إذ يقوم بمحاكاة الكائنات الحية في عملية البحث عن غذائها في الطبيعة, وتكون مثالية لحل مسائل الأمثلية غير الخطية Nonlinear Optimization Problems مع بعض المحددات. والثكل رقم (2) يمثل فئل بعض المجتمعات الطبيعية التي يحاكيها ذكاء السرب[11].

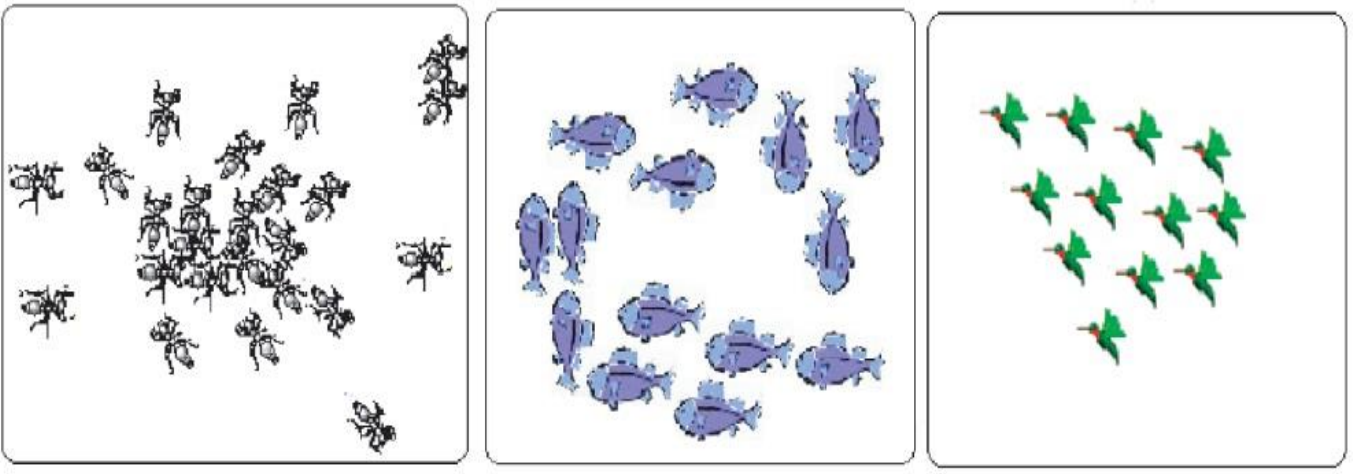

الثكل (2) يمثل بعض السلوك الجماعي لبعض أنواع الأسراب

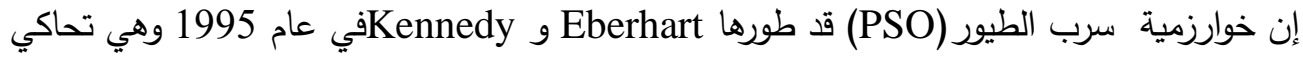
السلوك الاجتماعي لأسراب الطيور والأسماك التي تستخدمها هذه الأسراب لايجاد المأوى والمسكن ومصادر الغذاء أو موطن آخر ملائم لها, وتعد هذه الخوارزميه من أفضل الخوارزميات المعتمده في حل كثير من التقانات وأن

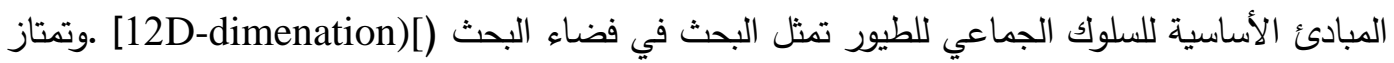
خوارزميته بما يأتي:

• التجانس Homogeneity: الطيور كلها لها أنموذج السلوك نغسه ويتحرك السرب بدون قائد.

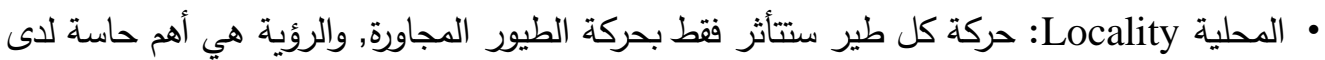

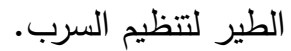

• تجنب التصادم Collision Avoidance: يقوم الطير بتجنب زملائه في السرب. • التمركز في السرب Flock Centering: يحاول الطير البقاء على مقربة من زملائه في السرب. • مطابقة السرعة Velocity Matching: يقوم الطير بمحاولة مجاراة سرعة زملائهِ في السرب [13]. 
في هذا البحث استخدمت خوارزمية أمثلية عناصر السرب في تحقيق جودة البرمجيات; إذ تقوم بأخذ عينة عشوائيه لمجموعة التدريب لتكوين نماذج مستندةعليها لعملية قياس نوعية الأداء حيث البيانات التي أخذت من فن وكالة NASA هي (pc1) والتي تحتوي على 22 مقياساً و 1109 وحدة, وأن هذه المقاييس تستخدم لوصف جودئ نودة

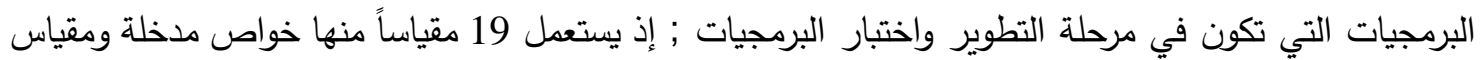
واحد يمثل الناتج الفعلي, وهذه المقاييس موضحة في الجدول (1).

الجدول(1) يمثل مجموعة المقاييس المستخدمة في البحث

\begin{tabular}{|c|c|c|c|c|c|}
\hline الوحدة 1109 & $\cdots \cdots$ & الوحدة 500 & ..... & الوحدة 1 & المقاييس \\
\hline 26 & $\ldots$. & 4 & $\ldots$. & 1.1 & Lines of code \\
\hline 18 & $\ldots .$. & 1 & $\ldots$. & 1.4 & Cyclomatic Complexity \\
\hline 13 & $\ldots .$. & 1 & $\ldots .$. & 1.4 & Essential Complexity \\
\hline 6 & $\ldots .$. & 1 & $\ldots .$. & 1.4 & Design Complexity \\
\hline 228 & $\ldots$. & 7 & $\ldots .$. & 1.3 & Program Length \\
\hline 1335.62 & $\ldots$. & 19.65 & $\ldots$. & 1.3 & Program Volume \\
\hline 0.03 & $\ldots .$. & 0.4 & $\ldots$. & 1.3 & Program Level \\
\hline 35.81 & $\ldots$. & 2.5 & $\ldots .$. & 1.3 & Difficulty Level \\
\hline 37.29 & $\ldots .$. & 7.86 & $\ldots .$. & 1.3 & Intelligent Content \\
\hline 47834.26 & $\ldots$. & 49.13 & $\ldots .$. & 1.3 & Programming Effort \\
\hline 0.45 & $\ldots$. & 0.01 & $\ldots .$. & 1.3 & Program Vocabulary \\
\hline 2657.46 & $\ldots .$. & 2.73 & $\ldots$. & 1.3 & Programming Time \\
\hline 26 & $\ldots$ & 4 & $\ldots$. & 2 & LOCode \\
\hline 0 & $\ldots .$. & 0 & $\ldots .$. & 2 & LOCodeAndComment \\
\hline 23 & $\ldots .$. & 5 & $\ldots .$. & 1.2 & number of unique operators \\
\hline 35 & $\ldots .$. & 2 & $\ldots .$. & 1.2 & number of unique operands \\
\hline 119 & $\cdots \cdots$ & 5 & $\ldots$. & 1.2 & $\begin{array}{c}\text { total occurrences of } \\
\text { operators }\end{array}$ \\
\hline 109 & ..... & 2 & $\cdots \cdot$ & 1.2 & $\begin{array}{l}\text { total occurrences of } \\
\text { operands }\end{array}$ \\
\hline 35 & $\ldots .$. & 1 & $\ldots .$. & 1.4 & BranchCount \\
\hline 0 & $\ldots$. & 0 & $\cdots \cdot$ & 0 & Defects \\
\hline
\end{tabular}

والخطوات المتبعة لتمثيل خوارزمية البحث تتلخص بالخطوات الآتية:

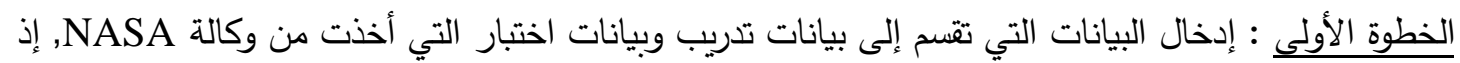

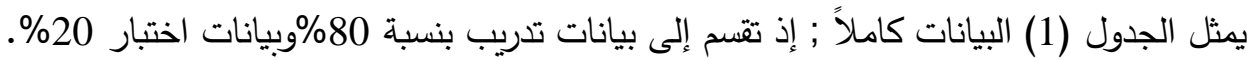
الخطوة الثانية :تحديد مصفوفة التي تستخدم لعمل استخلاص المواصفات [10*10] 
إذ يمثل الرقم 10 عدد الاختيارات التي سوف يختار قيمة واحدة منها, والرقم 16 يمثل عدد المواصفات التي

سيختار من بين 19 صفة .

الخطوة الثالثة : البدء بتتفيذ خوارزمية أمثلية سرب الطيور على بيانات التدريب بالاعتماد على 16 صفة عند الاختيار =1 من أصل 10 اختيارات التي حددت في الخطوة السابقة.

الخطوة الرابعة : تهيء السرع والمواقع بقيم ابتدائية حيث السرع تكون قيمها صفر في البداية والمواقع تمثل 16

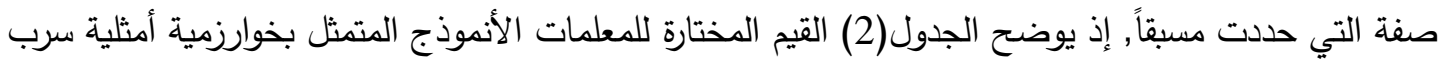
الطيور المدمجة بالثبكة العصبية ذات التغذية الجاهزة في ماتلاب, إذ تعتمد بصورة كبيرة على مجموعة المعلمات

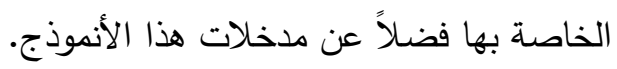

الجدول(2)مدخلات ومعلمات النموذج PSONN-SCQM

\begin{tabular}{|r|c|}
\hline PSONN-SCQM & Data \\
\hline PSO Iteration No. & 100 \\
\hline Swarm Size & 10 \\
\hline Inertia Weight(w) & 0.9 \\
\hline $\mathrm{c}_{1}$ & 0.2 \\
\hline $\mathrm{c}_{2}$ & 0.1 \\
\hline Rand1 & 0.2 \\
\hline Rand2 & 0.3 \\
\hline No. of Hidden Neurons & $\{16,4\}$ \\
\hline
\end{tabular}

الخطوة الخامسة:حساب دالة اللياقة للقيم لكل فرد( يمثل Pest 1 فضل موقع شخصي) بصورة ابتدائية التي تعتمد على دالة sphere, إذ تمثل الدالة المعادلة الاتية:

$f(x)=\sum_{i=1}^{n} x_{2}$

الخطوة السادسة: حساب قيمة gbest (أفضل موقع عام)رمقارنة قيمة دالة اللياقة مع أفضل موقع في السرب ككل,

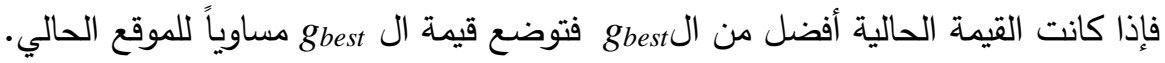
الخطوة السابعة : تحديث السرعة والموقع لكل فرد في السرب عند الدورة 1=t, وذلك بالاعتماد على المعادلتين $v_{i}^{k+1}=w^{k} v_{i}^{k}+c_{1} r_{1}^{k}\left(p_{i}^{k}-s_{i}^{k}\right)+c_{2} r_{2}^{k}\left(p_{g}^{k}-s_{i}^{k}\right)$

$s_{i}^{k+1}=s_{i}^{k}+v_{i}^{k+1}$

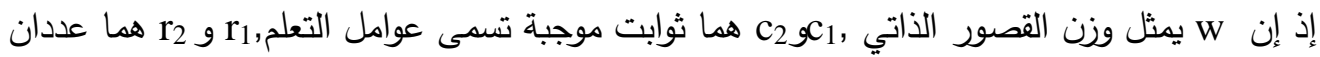

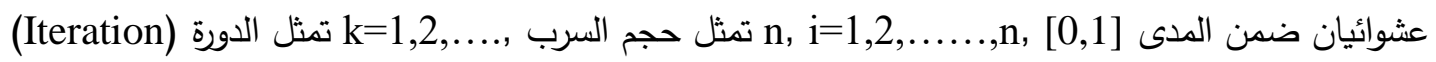

الخطوة الثامنة: حساب دالة اللياقة للقيم لكل فرد للقيم الجديدة.

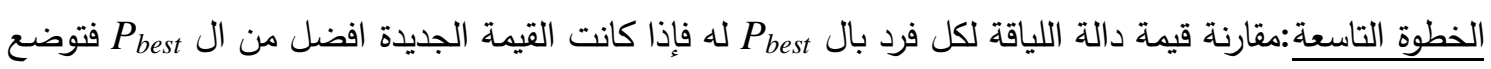
قيمة Pest مساوية للقيمة الجديدة وموقع ال Pest مساوياً للموقع الجديد في فضاء المسألة.

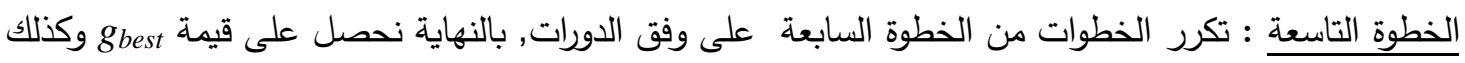
نقوم بخزن الأوزان الخاصة بالثبكة التي تكون خاصة بالمواصفات المحددة. 
الخطوة العاشرة : يكرر من الخطوة الثالثة على مواصفات محددة أخرى على وفق الاختيارات.

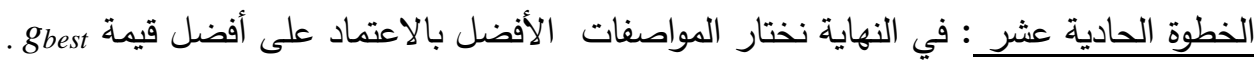
الخطوة الثانية عشر : تقييم الخوارزمية المقترحة بالاعتماد على المعايير الصحة.

والثكل(3) يمثل مخطط الفاعلية للخوارزمية المقترحة لقياس جودة البرمجيات التي تستند على استخلاص الخواص لمجموعة من الجسيمات التي تولد مصفوفة ثنائية الأبعاد بصورة عشوائية, إذ إنَ موقع كل فرد

Particle(i)

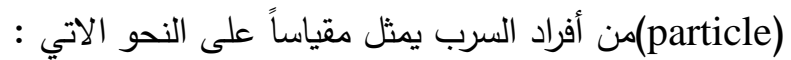

$=\left[p_{1} p_{2} p_{3} p_{4} p_{5} p_{6} p_{7} p_{8} p_{9} p_{10} p_{11} p_{12} p_{13} p_{14} p_{15} p_{16}\right]$ SwarmMatrix

$$
=\left[\begin{array}{c}
\text { Particle(1) } \\
\text { Particle(2) } \\
\cdot \\
\cdot \\
\cdot \\
\text { Particle(10) }
\end{array}\right]
$$

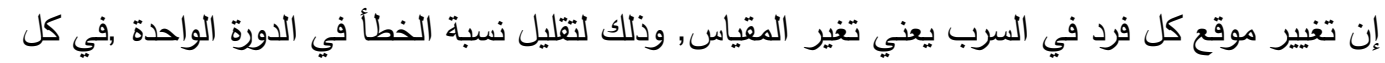

دورة تقوم أفرآد السرب بتغيير مواقعها بتحديث السرعة ومن ثم التحرك إلى الموقع الجديد الذي يمثل مقياساً جديداً

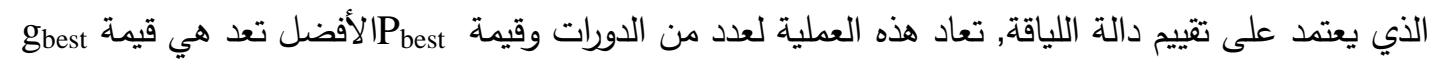

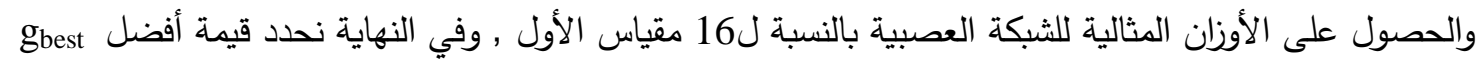
من بين 10قيم ل وال والتي سوف تحدد المقاييس 16 الأفضل من بين 10 احتمالات المحدد مسبقاً و الأوزان المثالية لشبكة العصبية روبعد أن يحدد ال16 مقياس الأفضل تحسب معايير الدقة لتصنيف جودة البرمجيات . 


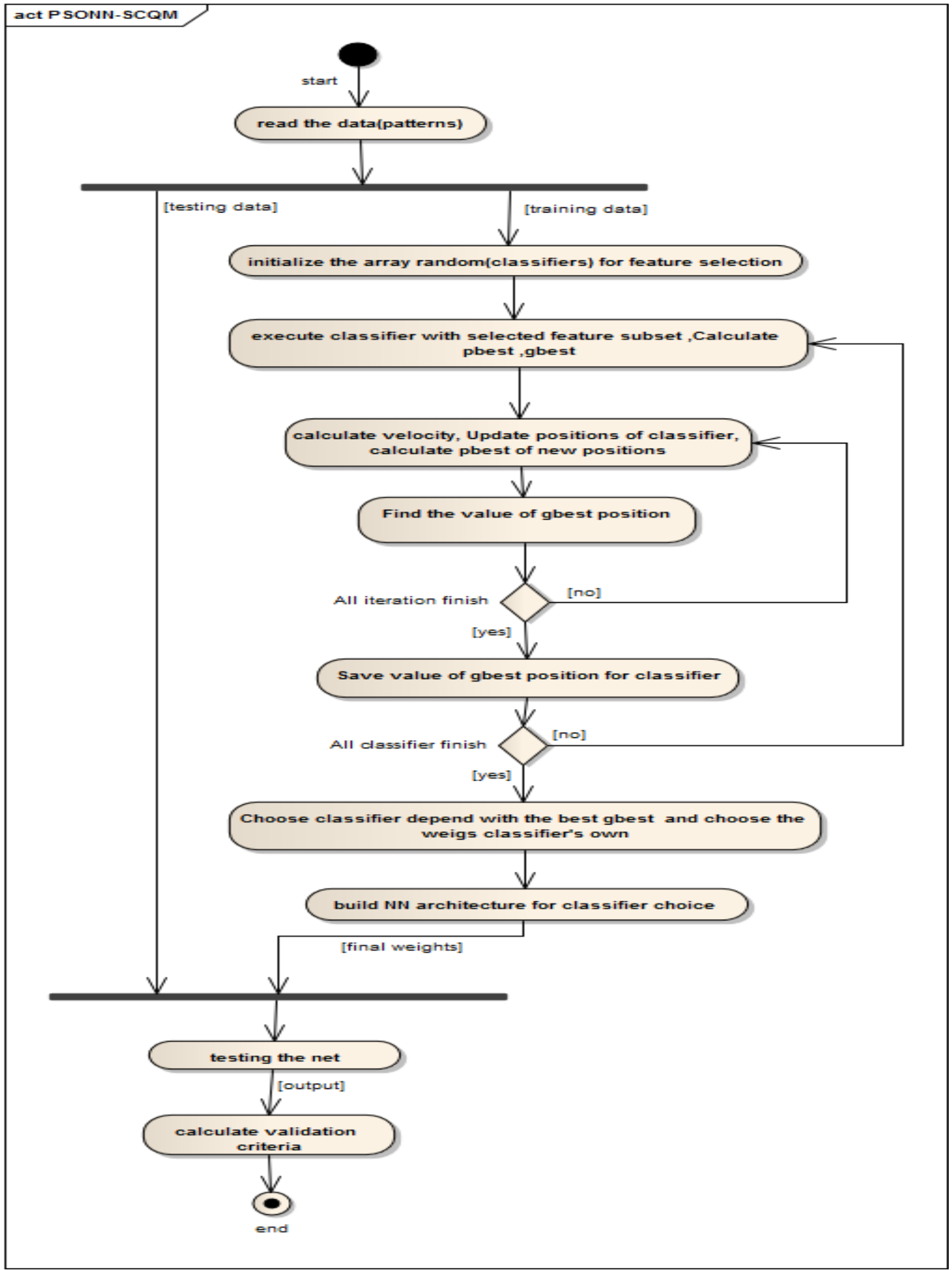

الشكل (3) يمثل مخطط الفاعلية للخوارزمية المقترحة

5. النتائج والاستنتاجات

طبقت الخوارزمية المقترحة باستخدام (Matlab R2011a) بالاعتماد على معايير الصحة التي تشمل حساب دقة التصنيف(Classification Accuracy)وحساب نسبة الخطأ (Error Rate)ومتوسط مربع الخطأ وجذر متوسط مربع الخطأ (Mean Square Error) 
الدقة من المئة ازدادت فرصة الوصول إلى الحل الأمثل التي تمثل الفرق بين القيمة الفعلية والقيمة المتوقعة ربينما نسبة الخطأ يحسب بالاعتماد على نسبة الدقة , متوسط مربع الخطأ يحسب مجموع مربع الفرق بين القيمة الفعلية والقيمة الناتجة على عدد القيم المستخدمة ,جذر متوسط مربع الخطأ يحسب الجذر التربيعي لمتوسط مربع الخطأ [15]وكما موضح بالجدول (3) يمثل نتائج تقييم الخوارزمية المقترحة لقياس جودة البرمجيات ,أما الثكل (4) فيمثل نتائج تقييم الخوارزمية المقترحة لقياس جودة البرمجيات. الجدول(3) يمثل نتائج تقييم الخوارزمية المقترحة لقياس جودة البردئ البرمجيات

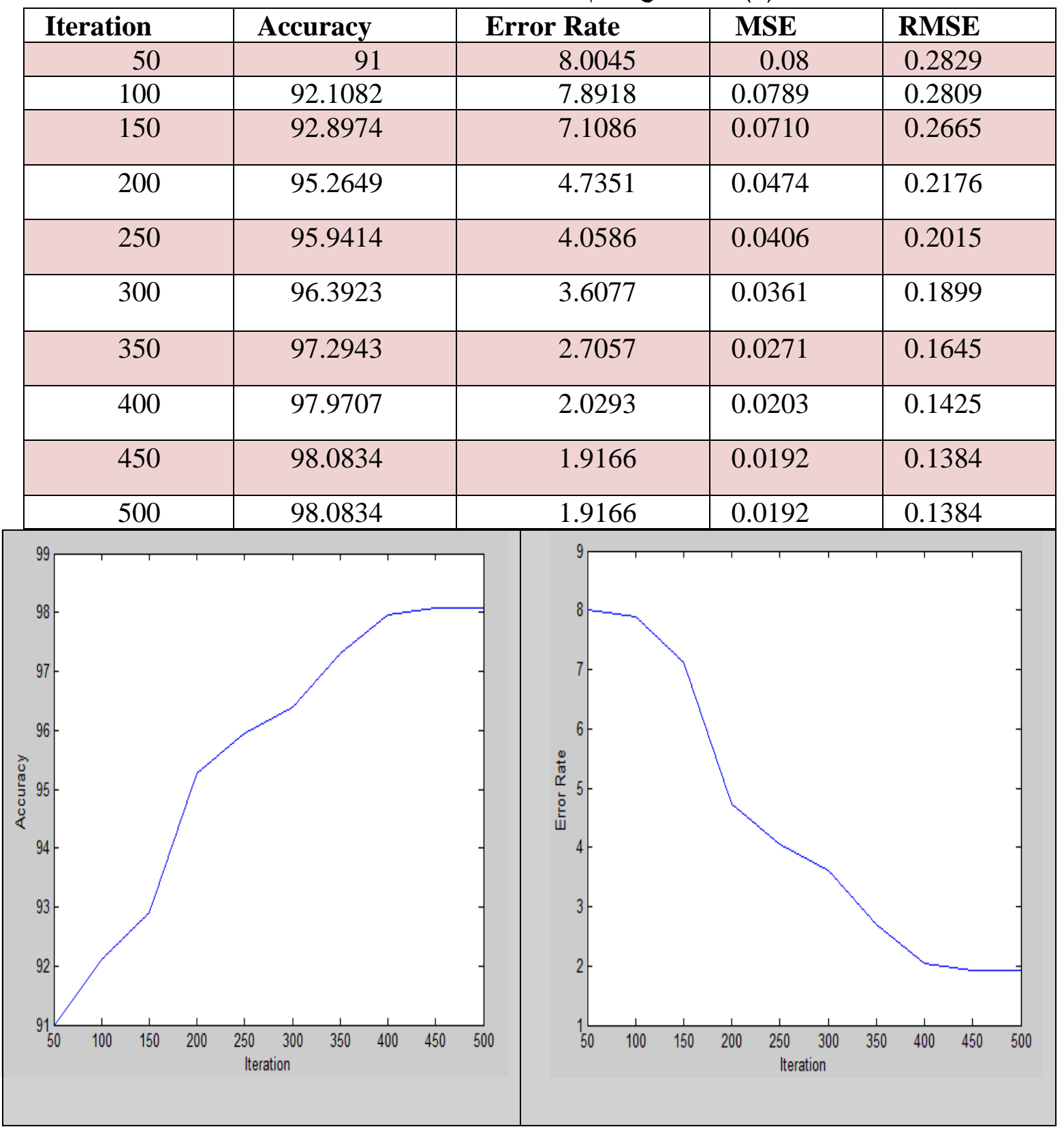




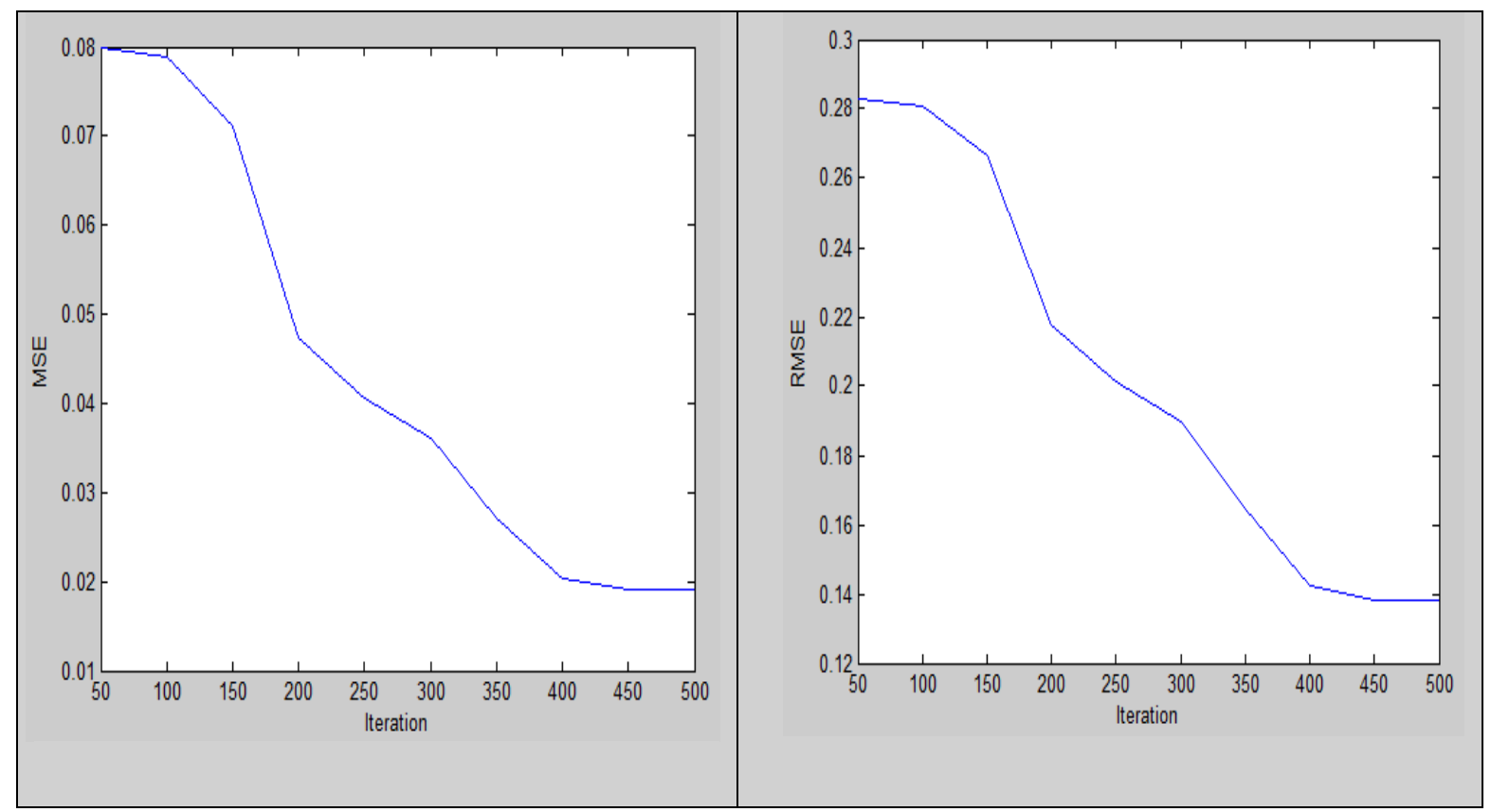

الثكل (4) يمثل نتائج تقييم الخوارزمية المقترحة لقياس جودة البرمجيات

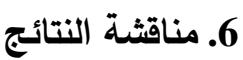

يتبين من استخدام خوارزمية أمثلية عناصر السرب التي هجنت مع الثبكة العصبية ذات الانتشار العكسي لاستخلاص الصفات التي تكونت عشوائياً في مصفوفة; وذلك لاختيار الصفات ذات القيم الأفضل وتحديد الأوزان المثالية للشبكة وبعدها تحسب قيمة المعايير الخاصة بقياس جودة البرنامج الذي حدد, وتبين من قيم معايير الدقة الموضحة في الجدول (3) أن قيمة الدقة في تزايد والمعايير الأخرى في نقصان عند استخدام الخوارزمية المقترحة ,

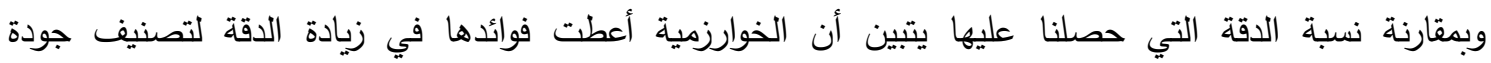
Combining "البرمجيات حيث توصلت إلى نسبة دقة 98\% وهي نسبة لم تصلها الطرائق المستخدمة في البحثه Particle Swarm Optimization based Feature Selection and Bagging for Software Defect Prediction 


\section{المصادر}

[1] IEEE Std 610.12., 1990, IEEE Standard Glossary of Software Engineering Terminology.

[2] Broy, M., (2011), " The Future of Software Engineering", S. Nanz (ed.), SpringerVerlag Berlin Heidelberg. ISBN 978-3-642-15186-6.

[3] Pressman, R., 2010, "Software Engineering: A Practitioner's Approach", $7^{\text {th }}$ Edition, McGraw-Hill, New York, USA, ISBN 978-0-07-337597-7

[4] Sommerville, I. , 2011 , "Software Engineering" , 9th Edition , Addison Wesley, ISBN-13: 978-0-13-703515-1

[5] S. Kim, H. Zhang, R. Wu and L. Gong, 2011 "Dealing with noise in defect prediction", Proceeding of the 33rd International Conference on Software engineering - ICSE '11, (), pp. 481-490.

[6] M. M. Kabir, M. Shahjahan and K. Murase, , (2012) "A new hybrid ant colony optimization algorithm for feature selection", Expert Systems with Applications, vol. 39, no. 3, pp. 3747-3763

[7] S. C. Yusta, (2009), "Different metaheuristic strategies to solve the feature selection problem", Pattern Recognition Letters, vol. 30, no. 5, pp. 525-534.

[8] Panovski , Gregor. ,(2008)," Product Software Quality ", MASTER'S THESIS, Department of Mathematics and Computing Science, TECHNISCHE UNIVERSITEIT EINDHOVEN.

[9] Fitzpatrick, Ronan. ,(1996)." Software Quality: Definitions and Strategic Issues", MSc Computing Science Advanced Research Module, Staffordshire University.

[10] Pressman, Roger S.,(2010)," Software Engineering: A Practitioner's Approach", 7th edition, McGraw-Hill, ISBN 978-0-07-337597-7, ISBN 0-07-337597-7.

[11] Mohd. Ehmer Khan. 2011, "Different Approaches to White Box Testing Technique for Finding Errors", International Journal of Software Engineering and Its Applications.

[12] Grosan, C., Abraham, A.,(2011), " Intelligence Systems", Springer, Verlag Berlin Heidelberg, ISBN 978-3-642-21003-7.

[13] Chang R, Lai L. , Wang J. and Kouh J. , 2007, "Intrusion Detection by Backpropagation Neural Networks with Sample-Query and Attribute-Query", Department of Engineering Science and Ocean Engineering, National Taiwan University, International Journal of Computational Intelligence Research. Vol.3, No. 1 .

[14] Gaitonde,V.,Karnik,S.,(2012),"Minimizing burr size in drilling using artificial neural network(ANN)-Particle swarm optimization(PSO) approach", Springer Science+ Business, J IntellManuf , doi: 10.1007/s10845-010-04815,Vol.23,pp.1783-1793.

[15] Saleem Basha, Dhavachelvan P., 2010, " Analysis of Empirical Software Effort Estimation Models ", (IJCSIS) International Journal of Computer Science and Information Security, Vol. 7, No. 3. 